\title{
Same-sign Tops: A Powerful Diagnostic Test for Models of New Physics
}

\author{
David Atwood, ${ }^{a}$ Sudhir Kumar Gupta, ${ }^{b}$ and Amarjit Soni ${ }^{c}$ \\ ${ }^{a}$ Dept. of Physics and Astronomy, Iowa State University, \\ Ames, IA 50011, USA \\ ${ }^{b}$ ARC Centre of Excellence for Particle Physics at the Terascale, School of Physics, Monash Uni- \\ versity, \\ Melbourne, Victoria 3800 Australia \\ ${ }^{c}$ Theory Group, Brookhaven National Laboratory, \\ Upton, NY, 11973, USA \\ E-mail: atwood@iastate.edu, Sudhir.Gupta@monash.edu, \\ adlersoni@gmail.com
}

\begin{abstract}
:
We study the connection between the same sign top (SST) and the top quark forwardbackward asymmetry $\left(\mathcal{A}_{F B}^{t}\right)$. We find that a large class of new physics (NP) models that have been proposed to account for the $\mathcal{A}_{F B}^{t}$ lead to SST quark production rate much larger than the observed rate at the LHC and consequently are severely constrained or ruled out. Our model independent, general, operator analysis shows that none of the tree-level selfconjugate flavor-changing operators are able to explain $\mathcal{A}_{F B}^{t}$ and simultaneously remain consistent with the same-sign top-quark production constraints from the LHC data.
\end{abstract}




\section{Contents}

1 Introduction 1

2 The General New Physics Operators 2

3 Correlation between the $A_{F B}^{t}$ and the $\sigma_{t t} \quad 5$

4 Numerical Analysis and Results $\quad 8$

$\begin{array}{lll}\text { 4.1 SM background } & 13\end{array}$

5 Conclusions $\quad 14$

\section{Introduction}

The forward-backward asymmetry of the top quark, $\mathcal{A}_{F B}^{t}$ as measured by the two Fermilab Tevatron experiments $[1,2]$ shows $\sim 2.5 \sigma$ deviation from the Standard model (SM) estimates [3, 4] and thus hints at some form of new physics in the top sector. On the other hand the measured top-pair production cross-section appears to be consistent with the SM prediction within errors. This creates tension in new physics models trying to explain the experimentally observed $\mathcal{A}_{F B}^{t}$ as the new interaction tends to modify the top-pair production cross-section as well.

Over the years, several speculations $[5,6]$ have been made to explain the observed value of $\mathcal{A}_{F B}^{t}$. Some key review papers can be found in the Refs. [6]. Axi-gluons, coloroctet, color-sextet, models with an extra vector boson(s) and/or scalar(s) etc. have been introduced to explain this effect. Depending upon whether the additional contribution to the $\mathcal{A}_{F B}^{t}$ is due to a s-channel exchange or due to a t-channel exchange, these suggestions fall into two broader categories. The former category of models require processes where the exchanged particle has flavor diagonal couplings to the SM-quarks. This is severely constrained from the direct searches on non-observation of any weakly coupling non-standard under- $\mathrm{TeV}$ resonance at the Tevatron or the LHC. The latter possibility requires processes in which an up- or charm-quark can transit into the top-quark or vice versa. Interestingly such (self-conjugate flavor-violating) interactions can also contribute in the production of the same-sign top pairs at the hadron collider and for this reason we focus on the same sign top pair production in this paper.

If these flavor-violating couplings of the top-quark are introduced to explain the $\mathcal{A}_{F B}^{t}$ then, at the LHC, pairs of same-sign top quarks can be produced via t-(and u-) channel exchange of scalar, vector or even tensor particles through the parton level process $u u \longrightarrow t t$ or $\bar{u} \bar{u} \longrightarrow \overline{t t}$. Same-sign top-pair production process is thus a very useful "smoking-gun" signature especially since it has little SM background and can be observed in the form of a 
pair of same-sign leptons in association with a b-jet pair due to semileptonic decays of the produced top-quarks. Recall that in models such as supersymmetry, due to the Majorana nature of the gluino, one expects to observe lots of such events when a pair of gluinos are produced and decay into tops in turn. The other known class of models where same-sign top production can be abundant are those where a boson with charge $( \pm 4 / 3)$ couples with tops and thus can give rise to SST-pair via s-channel resonance. It is to be noted that SUSY same-sign top events differ from our case in the sense that, SUSY events carries largee amount of missing tranverse energy due to missing lightest stable superparticles, and the event rates for both $t t$ and $\overline{t t}$ are the same, because top and anti-top decays of gluinos have the same rate. The other case mentioned is also different from ours in the sense that there we explicitly expect to observe a charge- $\pm 4 / 3$ resonance.

Note also that: (1) this production process has very little SM background as we briefly discuss in section 4, and, (2) the top quark is a self-analyser of its spin, several interesting observables can be constructed in order to test the models.

Thus the interplay among $\mathcal{A}_{F B}^{t}, \sigma_{t \bar{t}}$ and the $\sigma_{t t}$ seems very useful to explore. These three-fold experimental measurements should allow us to set up a strategy to single out the true new physics operators responsible for explaining the measured $\mathcal{A}_{F B}^{t}$.

Motivated with this, in the current paper we will establish some remedial measures for the $\mathcal{A}_{F B}^{t}$ by exploiting all possible set of self-conjugate flavor-changing operators of the top-quark which could be responsible for the observed deviation at the Tevatron.

Before closing this section, we would like to point out that because LHC is a symmetric

$p p$ machine, $\mathcal{A}_{F B}^{t}$ can not be defined at the LHC. However an analogus (parity-violating) observable to the $\mathcal{A}_{F B}^{t}$, called charge-asymmetry of the top-quark, $A_{C}$, can be still defined by the difference of number of $t \bar{t}$ in forward and backward rapidity region. Now since, at the LHC $t \bar{t}$ prodcution is dominated by the gluon-fusion, the $A_{C}$ is expected to be rather small, and therefore, it may not be an useful observable to study the flavor-violating operators under consideration and it is not our focus in this paper.

Organisation of the paper is as follows: In section 2 we will provide a detailed account of the most general form of operators that could contribute to the $\mathcal{A}_{F B}^{t}$. In section 3 we will discuss the correlation of the $\mathcal{A}_{F B}^{t}$ and the same-sign top-pair cross-section. We provide a detailed numerical analysis for each of the operators in light of the experimental measurements in section 4 . We conclude with the findings of this work in section 5 .

\section{The General New Physics Operators}

As reported in Ref. [1, 2], the independent measurements in the $l+j$, where $l=e^{ \pm}, \mu^{ \pm}$and $j$ is a jet, detection mode by the two experiments at the Fermilab Tevatron suggest $\mathcal{A}_{F B}^{t}$ to be $\mathcal{A}_{F B}^{t}(\mathrm{D} \varnothing)=0.19 \pm 0.065$, and, $\mathcal{A}_{F B}^{t}(\mathrm{CDF})=0.158 \pm 0.074$ which are clearly consistent with each other within experimental errors yielding a weighted average of $0.176 \pm 0.05$. This is somewhat higher than the the SM predictions of $0.058 \pm 0.009$ and $0.072_{-0.007}^{+0.011}$ at the NLO [3] and NLO + NNLL [4] levels respectively (note that at the tree level the asymmetry is identically zero). Thus the experimental results deviate by about $1.7 \sigma$ from the SM. Since the asymmetry in the high mass region, $\mathcal{A}_{F B}^{t, h i g h}$, tends to be a stronger discriminant 


\begin{tabular}{|c|c|c|}
\hline \hline Observable & Values & Experiment \\
\hline $\mathcal{A}_{F B}^{t}$ & $0.19 \pm 0.065$ & $\mathrm{D} \emptyset$ Collaboration [1] \\
& $0.158 \pm 0.074$ & CDF Collaboration [2] \\
& $0.176 \pm 0.05$ & Combined \\
\hline $\mathcal{A}_{F B}^{t, \text { low }}$ & $0.078 \pm 0.048$ & $\mathrm{D} \emptyset$ Collaboration [1] \\
& $-0.022 \pm 0.043$ & CDF Collaboration [2] \\
& $0.023 \pm 0.032$ & Combined \\
\hline $\mathcal{A}_{F B}^{t, h i g h}$ & $0.115 \pm 0.060$ & $\mathrm{D} \emptyset$ Collaboration $[1]$ \\
& $0.266 \pm 0.062$ & CDF Collaboration [2] \\
& $0.188 \pm 0.043$ & Combined \\
\hline$\sigma_{t \bar{t}}^{\text {Tevatron }}$ & $8.18_{-0.87}^{+0.98} \mathrm{pb}$ & $\mathrm{D} \emptyset$ Collaboration $[8]$ \\
\hline$\sigma_{l^{ \pm} l^{ \pm}}^{L H C}$ & $<1 \mathrm{fb}$ & ATLAS \& CMS Collaborations $[9]$ \\
\hline \hline
\end{tabular}

Table 1: Measured values of various observables used in our analysis; combined here means weighted averages.

of models, it may be useful to mention that $\mathcal{A}_{F B}^{t}$ in the high $m_{t t}$ region is experimentally (see Table 1) found to be $0.188 \pm 0.043$, whereas SM predicts $0.088 \pm 0.013$ [7] which is again off by $\sim 2.3 \sigma$.

The most general form of all possible sets of operators containing a color-neutral or colored scalar, a vector or a tensor can be written as follows,

$$
\begin{aligned}
Q_{A B}^{V_{s}} & =\left(\bar{u} \gamma^{\mu} P_{A} t\right)\left(\bar{u} \gamma_{\mu} P_{B} t\right), \\
Q_{A B}^{V_{o}} & =\left(\bar{u} \gamma^{\mu} P_{A} T^{a} t\right)\left(\bar{u} \gamma_{\mu} P_{B} T^{a} t\right), \\
Q_{A B}^{S_{s}} & =\left(\bar{u} P_{A} t\right)\left(\bar{u} P_{B} t\right), \\
Q_{A B}^{S_{o}} & =\left(\bar{u} P_{A} T^{a} t\right)\left(\bar{u} P_{B} T^{a} t\right), \\
Q_{A}^{T_{s}} & =\left(\bar{u} \sigma^{\mu \nu} P_{A} t\right)\left(\bar{u} \sigma_{\mu \nu} P_{A} t\right), \\
Q_{A}^{T_{o}} & =\left(\bar{u} \sigma^{\mu \nu} P_{A} T^{a} t\right)\left(\bar{u} \sigma^{\mu \nu} P_{A} T^{a} t\right),
\end{aligned}
$$

where, $P_{A}=\frac{1}{2}\left(a_{1}+a_{5} \gamma^{5}\right)$, and, $P_{B}=\frac{1}{2}\left(b_{1}+b_{5} \gamma^{5}\right)$. For $P_{A}=P_{L}, a_{1}=1=-a_{5}$ so for our model we keep $a_{1,5}$ and $b_{1,5}$ arbitrary so that we can study their dependence as well.

If we consider the case where $A B \in\{L L, R R, L R\}$ and $A \in\{L, R\}$ then there are 16 operators in this basis. Due to Fermi statistics of the identical t- and u-quarks these operators are not all linearly independent. Such a set consisting of 8 independent operators can be written as follows: 


$$
\begin{aligned}
Q_{1} & =-\frac{1}{3} Q_{L L}^{S_{s}}+Q_{L L}^{S_{o}} \\
Q_{2} & =\frac{2}{3} Q_{L L}^{S_{s}}+Q_{L L}^{S_{o}} \\
Q_{3} & =-\frac{1}{3} Q_{R R}^{S_{s}}+Q_{R R}^{S_{o}} \\
Q_{4} & =\frac{2}{3} Q_{R R}^{S_{s}}+Q_{R R}^{S_{o}} \\
Q_{5} & =-\frac{1}{3} Q_{R L}^{S_{s}}+Q_{R L}^{S_{o}} \\
Q_{6} & =\frac{2}{3} Q_{R L}^{S_{s}}+Q_{R L}^{S_{o}} \\
Q_{7} & =\frac{2}{3} Q_{L L}^{V_{s}}+Q_{L L}^{V_{o}} \\
Q_{8} & =\frac{2}{3} Q_{R R}^{V_{s}}+Q_{R R}^{V_{o}}
\end{aligned}
$$

In [10] a further condition is imposed that the operators are components of operators respecting the $S U(2) \times U(1)$ symmetry of the SM. These operators are linear combinations of $Q_{5-8}$ so $Q_{1-4}$ are eliminated if one imposes this further condition. In particular the uutt component of operators from that paper can be written in terms of the above as follows:

$$
\begin{gathered}
O_{R R}=Q_{8} \quad O_{L L}^{(1)}=Q_{7} \quad O_{L L}^{(3)}=Q_{7} \\
O_{L R}^{(1)}=Q_{6}-Q_{5} \quad O_{L R}^{(8)}=\frac{1}{3} Q_{6}-\frac{2}{3} Q_{5}
\end{gathered}
$$

Let us now consider the dimension 6 effective Lagrangian for $q \bar{q} \rightarrow t \bar{t}(q=u, d)$. Generically there are the 20 possible operators for each choice of $q$ which we may list according to a similar scheme as Eqn. 2.1:

$$
\begin{aligned}
{ }^{q} S_{A B}^{V_{i}} & =\left(\bar{q}^{a} \gamma^{\mu} P_{A} t^{b}\right)\left(\bar{t}^{c} \gamma_{\mu} P_{B} q^{d}\right) \\
{ }^{q} S_{A B}^{S_{i}} & =\left(\bar{q}^{a} P_{A} t^{b}\right)\left(\bar{t}^{c} P_{B} q^{d}\right), \\
{ }^{q} S_{A}^{T_{i}} & =\left(\bar{q}^{a} \sigma^{\mu \nu} P_{A} t^{b}\right)\left(\bar{t}^{c} \sigma_{\mu \nu} P_{A} q^{d}\right)
\end{aligned}
$$

here $q \in\{u, d\}$ and $a, b, c, d$ are color indices where $i \in\{s, o\}$ indicates whether the color structure is singlet $\left(\delta^{a b} \delta^{c d}\right)$ or octet $\left(T_{m}^{a b} T_{m}^{c d}\right)$.

The list constructed in this way contains some redundancy because some of the operators are self-conjugate while others appear in conjugate pairs. Enumerating the distinct operators we obtain the following: 


$$
\begin{aligned}
& { }^{q} S_{1}^{i}={ }^{q} S_{L R}^{S_{i}} \\
& { }^{q} S_{2}^{i}={ }^{q} S_{R L}^{S_{i}} \\
& { }^{q} S_{3}^{i}={ }^{q} S_{L L}^{V_{i}} \\
& { }^{q} S_{4}^{i}={ }^{q} S_{R R}^{V_{i}} \\
& { }^{q} S_{5}^{i}={ }^{q} S_{L R}^{V_{i}} \quad{ }^{q} S_{5}^{i \dagger}={ }^{q} S_{R L}^{V_{i}} \\
& { }^{q} S_{6}^{i}={ }^{q} S_{L L}^{S_{i}} \quad{ }^{q} S_{6}^{i \dagger}={ }^{q} S_{R R}^{S_{i}} \\
& { }^{q} S_{7}^{i}={ }^{q} S_{L}^{T_{i}} \quad{ }^{q} S_{7}^{i \dagger}={ }^{q} S_{R}^{T_{i}}
\end{aligned}
$$

Thus, operators ${ }^{q} S_{1-4}^{i}$ are self-conjugate and so must have real coefficients. The operators ${ }^{q} S_{5-7}^{i} ;{ }^{q} S_{5-7}^{i \dagger}$ are conjugate pairs. The coefficients of conjugate pairs must be conjugate; if they are complex then the model violates $\mathrm{CP}$.

Again, we can identify the operators which are consistent with $S U(2)$. Denoting by $u_{R}, d_{R}$ and $t_{R}$ the right handed components of the respective quarks and by $q_{L}=\left(\begin{array}{c}u_{L} \\ d_{L}\end{array}\right)$ and $Q_{L}=\left(\begin{array}{l}t_{L} \\ b_{L}\end{array}\right)$ the left handed light and heavy doublets respectively, these operators are:

$$
\begin{aligned}
& T_{1}^{i}=\left(\bar{u}_{R} \gamma^{\mu} t_{R}\right)\left(\bar{t}_{R} \gamma_{\mu} u_{R}\right)={ }^{u} S_{4} \\
& T_{2}^{i}=\left(\bar{u}_{R} Q_{L}\right)\left(\bar{Q}_{L} u_{R}\right)={ }^{u} S_{1}+\ldots \\
& T_{3}^{i}=\left(\bar{u}_{R} \gamma^{\mu} t_{R}\right)\left(\bar{Q}_{L} \gamma_{\mu} q_{L}\right)={ }^{u} S_{4}+\ldots \\
& T_{4}^{i}=\left(\bar{q}_{L} \gamma^{\mu} Q_{L}\right)\left(\bar{Q}_{L} \gamma_{\mu} q_{L}\right)={ }^{u} S_{3}+{ }^{d} S_{3}+\ldots \\
& T_{5}^{i}=\left(\bar{q}_{L} \gamma^{\mu} \tau^{i} Q_{L}\right)\left(\bar{Q}_{L} \gamma_{\mu} \tau^{i} q_{L}\right)={ }^{u} S_{3}-{ }^{d} S_{3}+\ldots \\
& T_{6}^{i}=\left(\bar{q}_{L} \gamma^{\mu} t_{R}\right)\left(\bar{t}_{R} \gamma_{\mu} q_{L}\right)={ }^{u} S_{2}+{ }^{d} S_{2} \\
& T_{7}^{i}=\left(\bar{d}_{R} \gamma^{\mu} t_{R}\right)\left(\bar{t}_{R} \gamma_{\mu} d_{R}\right)={ }^{d} S_{4} \\
& T_{8}^{i}=\left(\bar{d}_{R} Q_{L}\right)\left(\bar{Q}_{L} d_{R}\right)={ }^{d} S_{1}+\ldots \\
& T_{9}^{i}=\left(\bar{d}_{R} \gamma^{\mu} t_{R}\right)\left(\bar{Q}_{L} \gamma_{\mu} d_{L}\right)={ }^{d} S_{4}+\ldots
\end{aligned}
$$

The ellipses indicates additional terms which do not contribute to $\bar{q} q \rightarrow \bar{t} t$. Thus only operators ${ }^{q} S_{1-5}^{i}$ are consistent with $S U(2)$ while ${ }^{q} S_{6-7}^{i}$ are not. The only potentially CP violating operator consistent with $S U(2)$ is therefore ${ }^{q} S_{5}^{i}$.

\section{Correlation between the $A_{F B}^{t}$ and the $\sigma_{t t}$}

One key feature of analyzing new physics contribution to a process in terms of an effective Lagrangian is that as the mass scale becomes large, only the lowest dimension terms will contribute. This offers the prospect of reducing all possible models to a finite number of coefficients of operators which can, in principle, be associated with different signals. For 
this to work it is important to quantify how small the scale of new physics can be for this parameterization to be accurate.

In Figure 1 we address this issue by comparing the t-channel exchange of either a massive scalar or vector particle, $X$, with the dimension 6 approximation to that interaction.

In Figure 2 we apply such a comparison to the case of same sign top production. In the solid lines we consider a model where the amplitude is equal to

$$
\frac{g^{2}}{t-M_{X}^{2}} \bar{u} \Gamma_{1} t \quad \bar{u} \Gamma_{2} t
$$

where $\Gamma_{i}$ (which may contain Lorentz or color indices) determines the nature of the exchanged entity. As indicated several choices for $\Gamma_{i}$ are considered in this figure.

The dimension 6 model for such a general particle exchange is recovered by setting $t \rightarrow 0$ in the above expression. The cross section for this result is given by the dashed line. In both cases we have set $g_{X}=1$ for the normalization for the cross sections which are taken at $\sqrt{s}=7 \mathrm{TeV}$ and include the branching ratio of tops decaying leptonicaly. In addition we have applied the basic cuts that the transverse momentum of a lepton or a jet is $P_{T}>25 \mathrm{GeV}$ while the rapidity of these objects is $|\eta|<2.7$ and their separation is $\Delta R>0.4$. A missing transverse energy cut of $\mathbb{E}_{T}>30 \mathrm{GeV}$ is also applied. The dimension 6 limit appears to agree with the model within $\lesssim 30 \%$ when the mass of the exchange particle $M_{X} \geq 2 \mathrm{TeV}$. Below that point the two models begin to diverge. Note that since there is only one amplitude this conclusion is independent of the assumed value of $g_{X}$.

In Figure 1 we carry out the same exercise for the contribution of various t-channel exchanged objects to the forward-backward asymmetry of top-quark production. In this case the dimension 6 model agrees to a good approximation $(\lesssim 20 \%)$ with the exchange model when $M_{X}>1 \mathrm{TeV}$.

For cases which could provide an explanation for the observed value of $\mathcal{A}_{F B}$ a more general class of models must be considered which includes operators of dimension higher than 6 . This will necessarily constrain somewhat the generality of the conclusions we can reach.

In the cases we are considering, all the higher dimensional operators which can contribute at tree level are derived from the dimension 6 set by the insertion of pairs of covariant derivatives. In momentum space this means that the Feynman rules for these operators are modified by a form factor which depends on the kinematics of the scattering. Without loss of generallity we can write such a form factor as a function of $t$ and $u$ where in the reaction $u\left(p_{1}\right) u\left(p_{2}\right) \rightarrow t\left(p_{3}\right) t\left(p_{4}\right)$ or $u\left(p_{1}\right) \bar{u}\left(p_{2}\right) \rightarrow t\left(p_{3}\right) \bar{t}\left(p_{4}\right)$ we define $t=\left(p_{1}-p_{3}\right)^{2}$ and $u=\left(p_{1}-p_{4}\right)^{2}$.

If we generalize the operators in Eqn. 2.2 we need to take into account the correct symmetry under the exchange of identical fields. Thus for each operator we introduce two form factors $q_{i}^{+}(t, u)$ and $q_{i}^{-}(t, u)$ which have the symmetries: $q_{i}^{+}(t, u)=q_{i}^{+}(u, t)$ and $q_{i}^{-}(t, u)=-q_{i}^{-}(u, t)$. In terms of these symmetric and ant-symmetric functions we define: 


$$
\begin{aligned}
q^{s}(t, u) & =\frac{2}{3} q_{i}^{+}(t, u)-\frac{1}{3} q_{i}^{-}(t, u) \\
q^{s \prime}(t, u) & =-\frac{1}{3} q_{i}^{+}(t, u)+\frac{2}{3} q_{i}^{-}(t, u) \\
q^{o}(t, u) & =\frac{1}{2}\left(q_{i}^{+}(t, u)+q_{i}^{-}(t, u)\right)
\end{aligned}
$$

The generalization of these operators is thus:

$$
\begin{aligned}
& \hat{Q}_{1}=q_{1}^{s \prime}(t, u) Q_{L L}^{S_{s}}+q_{1}^{o}(t, u) Q_{L L}^{S_{o}} \\
& \hat{Q}_{2}=q_{2}^{s}(t, u) Q_{L L}^{S_{s}}+q_{2}^{o}(t, u) Q_{L L}^{S_{o}} \\
& \hat{Q}_{3}=q_{3}^{s \prime}(t, u) Q_{R R}^{S_{s}}+q_{3}^{o}(t, u) Q_{R R}^{S_{o}} \\
& \hat{Q}_{4}=q_{4}^{s}(t, u) Q_{R R}^{S_{s}}+q_{4}^{o}(t, u) Q_{R R}^{S_{o}} \\
& \hat{Q}_{5}=q_{5}^{s \prime}(t, u) Q_{R L}^{S_{s}}+q_{5}^{o}(t, u) Q_{R L}^{S_{o}} \\
& \hat{Q}_{6}=q_{6}^{s}(t, u) Q_{R L}^{S_{s}}+q_{6}^{o}(t, u) Q_{R L}^{S_{o}} \\
& \hat{Q}_{7}=q_{7}^{s}(t, u) Q_{L L}^{V_{s}}+q_{7}^{o}(t, u) Q_{L L}^{V_{o}} \\
& \hat{Q}_{8}=q_{8}^{s}(t, u) Q_{R R}^{V_{s}}+q_{8}^{o}(t, u) Q_{R R}^{V_{o}}
\end{aligned}
$$

Likewise in the case of $q \bar{q} \rightarrow t \bar{t}$ we can generalize the operators given in Eqn. 2.5 by replacing their coefficients in the effective lagrangian with a form factor depending on $\mathrm{s}$ and t. In this case, there is no constraint arising from the fermi symmetry of identical particles. We denote the form factors which apply to the operator ${ }^{q} S_{j}^{i}$ (see eq. 5 ) as ${ }^{q} s_{j}^{i}(s, t)$.

Models which are well approximated by the dimension 6 Lagrangian correspond to cases where the form factors are constant. Without considering fully general form factors, we consider the class of models where both $u u \rightarrow t t$ and $u \bar{u} \rightarrow t \bar{t}$ are generated by a the exchange of a single species of particle, $X$, in the t-channel of mass $M_{X}$ where $X$ may be either a scalar or a vector and also could be either a color singlet or an octet.

In general the form factor for a $u u \rightarrow t t$ process would be:

$$
q_{i}^{ \pm}=C_{u t}^{2}\left(\frac{1}{t-M_{X}^{2}} \pm \frac{1}{u-M_{X}^{2}}\right)
$$

where the choice of $i$ depends on the spin and helicity structure of the coupling of $X$ to the quarks and $C_{a b}$ is a matrix in flavor space of the couplings of $X$ to quarks. Likewise in the case of $u \bar{u} \rightarrow t \bar{t}$ the form factor will be

$$
{ }^{u} s_{j}^{i}=C_{u t} C_{t u}\left(\frac{1}{t-M_{X}^{2}}\right)
$$

We will further assume that the coupling matrix is self-conjugate, in particular, it has the symmetry that $\left|C_{u t}\right|=\left|C_{t u}\right|$. Therefore we define $\left|C_{u t}\right|^{2}=g_{X}^{2}$. 
With these assumptions then, a given type of $X$ will generate form factors for both the processes $u u \rightarrow t t$ (see, Fig. 3) and $u \bar{u} \rightarrow t \bar{t}$. In the limit of large $M_{X}$ this model will correspond to the dimension 6 Lagrangian with coefficients $-g_{X}^{2} / M_{X}^{2}$.

For our purposes we considered the following six realistic possibilities to each of these with a coupling constant $g_{X}$;

- $P_{A}=P_{L}=P_{B}$,

- $P_{A}=P_{L}, P_{B}=P_{R}$ or vice-versa,

- $P_{A}=P_{R}=P_{B}$,

- $P_{A}=I=P_{B}$,

- $P_{A}=I, P_{B}=\gamma_{5}$ or vice-versa, and,

- $P_{A}=\gamma_{5}=P_{B}$.

This thus leads to $6 \times 6=36$ independent cases for our analysis. Apart from the SM parameters, every case will have the mass of the exchanged particle $M_{X}$ and the flavorviolating coupling constant $g_{X}$.

The SM and NP contributions to the top-quark forward-backward asymmetries $\mathcal{A}_{F B}^{t, S M}$ and $\mathcal{A}_{F B}^{t, N P}$ are correlated to the $t \bar{t}$ cross-section in the following manner

$$
\begin{aligned}
\mathcal{A}_{F B}^{t, T o t a l} & =\left(\frac{\sigma_{t \bar{t}}^{S M}}{\sigma_{t \bar{t}}^{T o t a l}}\right) \mathcal{A}_{F B}^{t, S M}+\left(\frac{\sigma_{t \bar{t}}^{N P}}{\sigma_{t \bar{t}}^{T o t a l}}\right) \mathcal{A}_{F B}^{t, N P}, \\
\mathcal{A}_{F B}^{t, S M} & =\frac{\sigma_{t \bar{t}}^{S M}(\cos \theta>0)-\sigma_{t \bar{t}}^{S M}(\cos \theta<0)}{\sigma_{t \bar{t}}^{S M}}, \\
\mathcal{A}_{F B}^{t, N P} & =\frac{\sigma_{t \bar{t}}^{N P}(\cos \theta>0)-\sigma_{t \bar{t}}^{N P}(\cos \theta<0)}{\sigma_{t \bar{t}}^{N P}} ;
\end{aligned}
$$

where $\sigma_{t \bar{t}}^{\text {Total }}=\sigma_{t \bar{t}}^{S M}+\sigma_{t \bar{t}}^{N P}, \theta$ being the angle of the top quark in the $t \bar{t}$ centre-of-mass frame. Note also that the superscript NP (for new physics) here means any specific beyond the SM operator.

\section{Numerical Analysis and Results}

To gain further insight, in our work, rather than concentrating on the total $\mathcal{A}_{F B}^{t}$ we will analyse the integrated $\mathcal{A}_{F B}^{t}$ in the low top-pair invariant mass region $(<450 \mathrm{GeV})$ and in the high top-pair invariant mass region $(\geq 450 \mathrm{GeV})$, i.e. $\mathcal{A}_{F B}^{t, l o w}$ and $\mathcal{A}_{F B}^{t, h i g h}$ respectively. The measurements of each of these are listed in Table 1. Thus at least the following four observables need to be satisfied by any operator that aims to provide an experimentally viable solution to the $\mathcal{A}_{F B}^{t}$,

(a) top-pair production cross-section, $\sigma_{t \bar{t}}$,

(b) the integrated asymmetry in low $m_{t \bar{t}}(<450 \mathrm{GeV})$ region, $\mathcal{A}_{F B}^{t, l o w}$, 

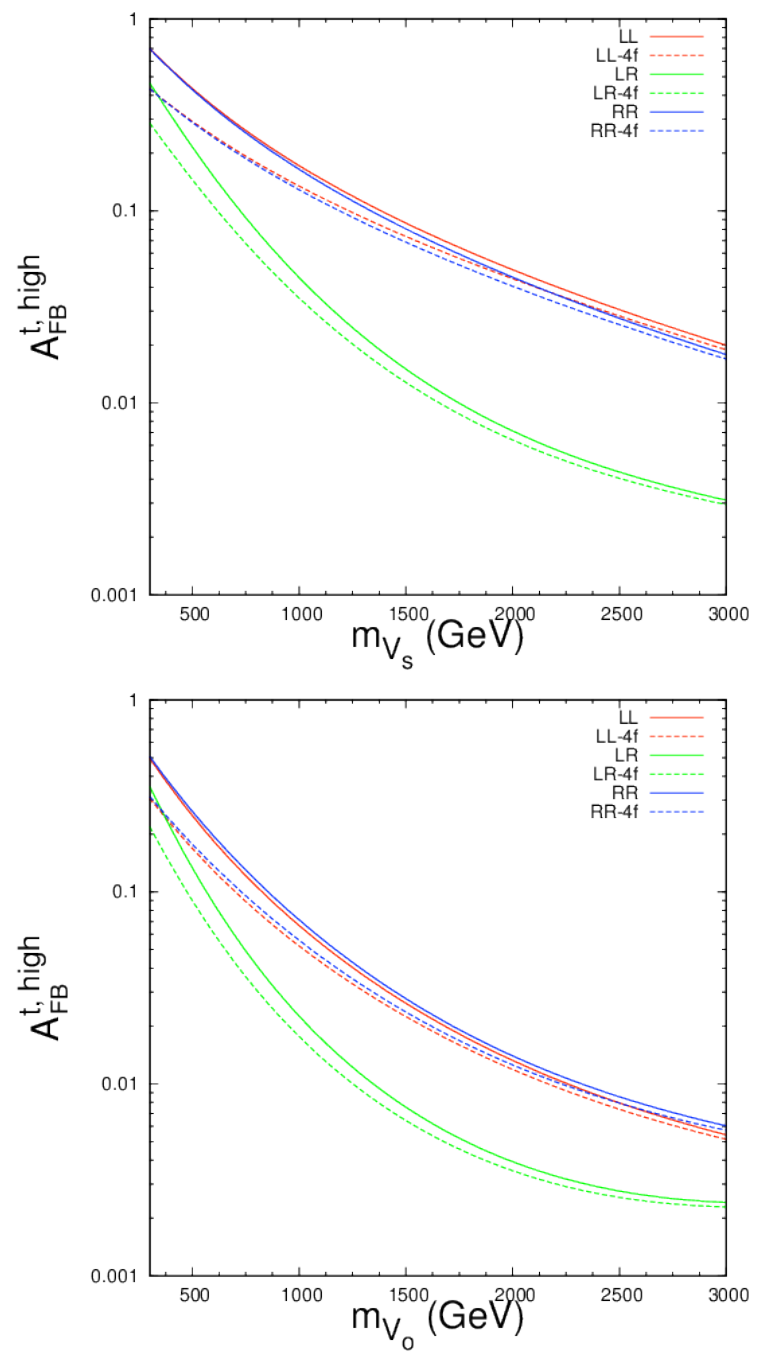

Figure 1: Tevatron high mass forward-backward asymmetry, $\mathcal{A}_{F B}^{t, h i g h}$, vs $M_{X}$ for various cases. In all cases, the solid lines show the exchange including a t-channel form factor while the dashed lines show the dimension four fermi operator without such a form factor. The upper graphs shows cases which are color singlet while the lower case shows color octet. In both graphs the red lines indicate LL vector couplings, the blue lines indicate RR vector couplings and the green line indicates LR vector couplings. In all cases $g_{X}=1$.

(c) the integrated asymmetry in high $m_{t \bar{t}}(\geq 450 \mathrm{GeV})$ region, and, $\mathcal{A}_{F B}^{t, h i g h}$, and,

(d) same-sign top pair cross-section at the LHC, $\sigma_{t t}$.

For our numerical analysis we implemented the aforementioned model into the package MadGraph5 [11] using FeynRules [12] and reproduced some of the results in Ref. [10] for checks. To evaluate the parton densities we use CTEQ6L1 [13]. The renormalisation scale $\mu_{R}$, and the factorisation scale, $\mu_{F}$ are fixed at $\mu_{R}=\sqrt{\hat{s}}=\mu_{F}$. We scan the following ranges of the $M_{X}-g_{X}$ parameter space: $g_{X}$ between $10^{-2}$ to 1 and $M_{X}$ between $0.2 \mathrm{TeV}$ to $4 \mathrm{TeV}$.

We analyse each operator individually in the following three steps: In order to find the 


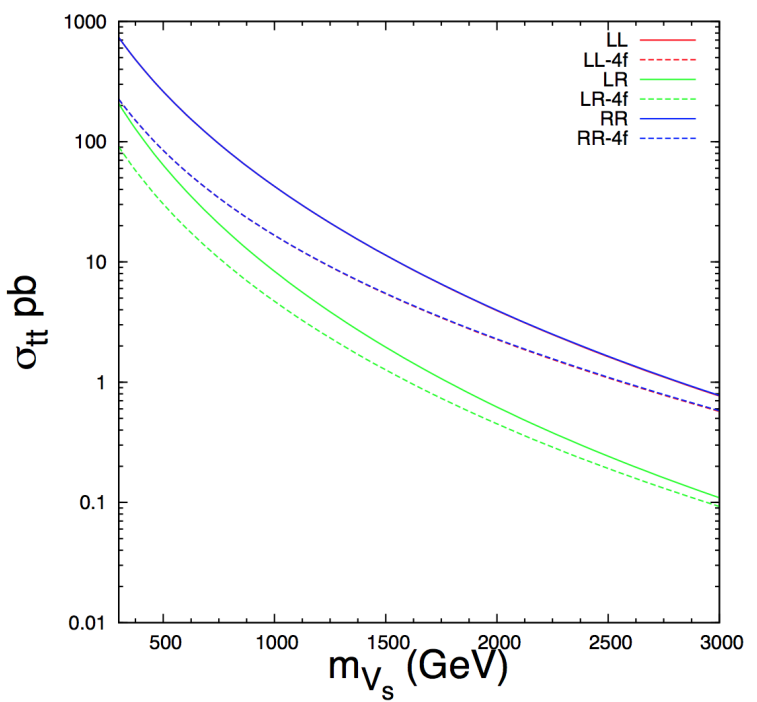

Figure 2: The same-sign top pair cross section at the LHC with $\sqrt{s}=7 \mathrm{TeV}$ for for various color singlet vector cases. In all cases, the solid lines show the exchange including a t-channel form factor while the dashed lines show the dimension four fermi operator without such a form factor. The red lines indicate $L L$ vector couplings, the blue lines indicate RR vector couplings and the green line indicates $L R$ vector couplings. In all cases $g_{X}=1$. Note that the $\mathrm{LL}$ and RR curves fall on top of each other since they are related by parity.

parameter space favoured by various operators as mentioned in Eqns. 2.1, we first ensure that for each of the allowed parameter space point the new physics contribution to the $t \bar{t}$ remains below $30 \%$ of the SM $t \bar{t}$ cross-section, To implement the constraints due to low and high mass region integrated asymmetries we first combine their measured values by the $\mathrm{CDF}$ and $\mathrm{D} \emptyset$ into corresponding weighted averages as mentioned in Table 1 and then allow only those parameter space points for which the theoretical asymmetries due to each of the two $m_{t \bar{t}}$ regions are within $2 \sigma$ standard deviation from the measured values.

Finally we enforce that the same-sign leptons (which arise due to the leptonic decays of the produced pair of same-sign tops) cross-section also remains within the LHC non-observation limits [9] for the current data on such events.

In order to demonstrate the dependence of $\mathcal{A}_{F B}^{t}$ in Figs. 1 we plot $\mathcal{A}_{F B}^{\text {thigh }}$ for the color singlet and octet vector cases with $L L, L R$ and $R R$ operators for a fixed values of $g_{X}=1$. Clearly a wide $M_{X}$ range below $\lesssim 2 \mathrm{TeV}$ is capable of producing the desired asymmetry for LL and RR singlet vector cases. This range becomes relatively narrower $(\lesssim 1 \mathrm{TeV})$ for the mixed case with LR couplings. The cases with octet vectors are more restricted (i.e. only $M_{X} \sim$ several hunders of GeVs are allowed) because their contribution to the $\mathcal{A}_{F B}^{t}$ is suppressed by the color-factor of $3 / 8$.

To demonstrate how strong these individual constraints can be, in Fig. 4 we show some of vector cases where at least some of the parameter space is allowed (marked with diagonal lines) after imposing the requirement that the new physics contribution to the total $t \bar{t}$ cross-section at the Tevatron should not exceed $30 \%$ and also the results must be consistent with the measured $\mathcal{A}_{F B}^{t}$ at $2 \sigma$ level. Thus we find that in order to produce 


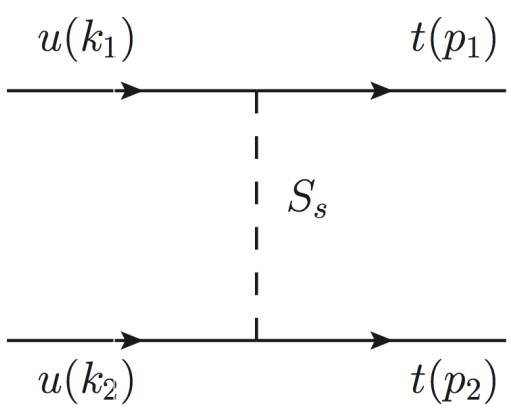

(a)

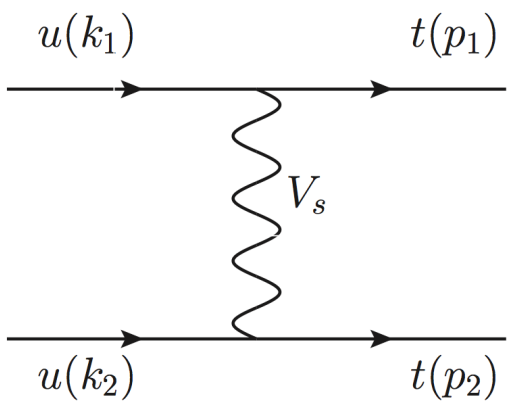

(c)

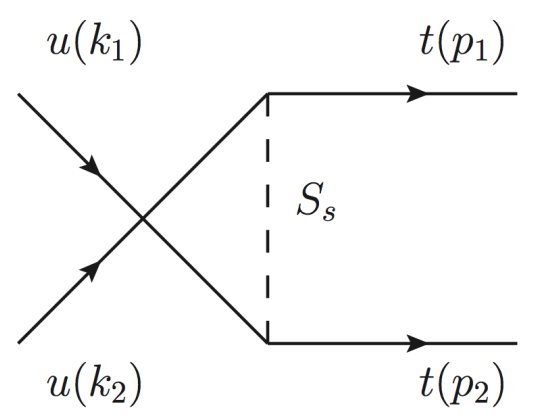

(b)

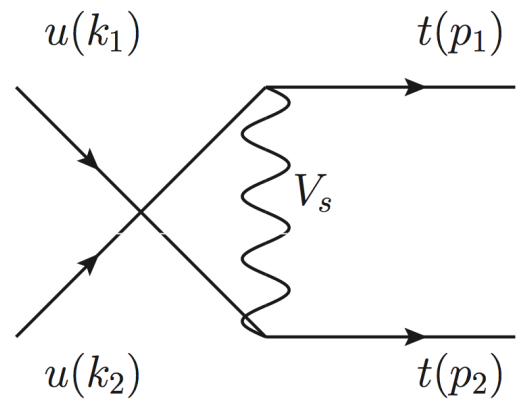

(d)

Figure 3: Representative parton level Feynman diagrams for the same-sign top pair production at the LHC via a scalar-singlet $\left(S_{s}\right)$ exchange (Figs. (a) and (b)), and, a vector-singlet $\left(V_{s}\right)$ exchange (Figs. (c) and (d)).

sufficiently large $\mathcal{A}_{F B}^{t}$ consistent with the experiment, $g_{X}$ must be sufficiently large and/or $M_{X}$ must be small while at the same time smaller correction in $\sigma_{t \bar{t}}$ requires the $g_{X}$ to be small and/or $M_{X}$ larger which essentially means that the two constraints push the $g_{X}-M_{X}$ from the two extremes.

In Fig. 2 we plot the same-sign top-pair cross-sections for the $\mathrm{LHC}$ at $7 \mathrm{TeV}$ for fixed values of $g_{X}=1$. To this end it is worth emphasising that for fixed $M_{X},\left.\sigma_{t t}\left(g_{X}\right)\right|_{M_{X}}=$ $g_{X}^{4} \times\left.\sigma_{t t}\left(g_{X}=1\right)\right|_{M_{X}}$. Also we expect the efficiency of cuts to be the same for fixed $M_{X}$. Clearly an order of magnitude suppression in $g_{X}$ would mean four orders of reduction in the same-sign top-pair event rate for the fixed $M_{X}$.

The aforementioned production processes give rise to a pair of same-sign leptons associated with a b-jet pair when both the produced tops decay leptonically in turn. Because so far LHC did not observe such events [9], we therefore would like to analyse the above couplings in light of the LHC non-observation constraints for this process. 


\begin{tabular}{|l|l|l|l|l|}
\hline \hline Operator & \multicolumn{5}{|c|}{ Observables } \\
\hline & $\frac{\delta \sigma_{t \bar{t}}}{\sigma_{t \overline{S M}}^{S M}}$ & $+\chi_{A_{F B}^{t, l o w}}$ & $+\chi_{A_{F B}^{t, h i g h}}$ & $+\sigma_{l^{ \pm} l^{ \pm}}$ \\
\hline & Case 1: Singlet Scalar \\
\hline \multicolumn{5}{|c|}{} \\
\hline$Q_{L L}^{S_{s}}$ & $\checkmark$ & $\checkmark$ & $\checkmark$ & $\times$ \\
\hline$Q_{L R}^{S_{s}}$ & $\checkmark$ & $\checkmark$ & $\times$ & $\times$ \\
\hline$Q_{R R}^{S_{s}}$ & $\checkmark$ & $\checkmark$ & $\checkmark$ & $\times$ \\
\hline$Q_{11}^{S_{s}}$ & $\checkmark$ & $\checkmark$ & $\times$ & $\times$ \\
\hline$Q_{15}^{S_{s}}$ & $\checkmark$ & $\checkmark$ & $\times$ & $\times$ \\
\hline$Q_{55}^{S_{s}}$ & $\checkmark$ & $\checkmark$ & $\times$ & $\times$ \\
\hline \hline \multicolumn{5}{|c|}{ Case 2: Octet Scalar } \\
\hline \hline$Q_{L L}^{S_{o}}$ & $\checkmark$ & $\checkmark$ & $\times$ & $\times$ \\
\hline$Q_{L R}^{S_{o}}$ & $\checkmark$ & $\checkmark$ & $\times$ & $\times$ \\
\hline$Q_{R R}^{S_{o}}$ & $\checkmark$ & $\checkmark$ & $\times$ & $\times$ \\
\hline$Q_{11}^{S_{o}}$ & $\checkmark$ & $\checkmark$ & $\times$ & $\times$ \\
\hline$Q_{15}^{S_{o}}$ & $\checkmark$ & $\checkmark$ & $\times$ & $\times$ \\
\hline$Q_{55}^{S_{o}}$ & $\checkmark$ & $\checkmark$ & $\times$ & $\times$ \\
\hline \hline \multicolumn{5}{|c|}{ Case 3: Singlet Vector } \\
\hline \hline$Q_{L L}^{V_{s}}$ & $\checkmark$ & $\checkmark$ & $\checkmark$ & $\times$ \\
\hline$Q_{L R}^{V_{s}}$ & $\checkmark$ & $\checkmark$ & $\times$ & $\times$ \\
\hline$Q_{R R}^{V_{s}}$ & $\checkmark$ & $\checkmark$ & $\checkmark$ & $\times$ \\
\hline$Q_{11}^{V_{s}}$ & $\checkmark$ & $\checkmark$ & $\checkmark$ & $\times$ \\
\hline$Q_{15}^{V_{s}}$ & $\checkmark$ & $\checkmark$ & $\times$ & $\times$ \\
\hline$Q_{55}^{V_{s}}$ & $\checkmark$ & $\checkmark$ & $\checkmark$ & $\times$ \\
\hline \hline & $\checkmark$ & $\times$ \\
\hline \hline$Q_{L L}^{V_{o}}$ & $\checkmark$ & $\checkmark$ & $\times$ & $\times$ \\
\hline$Q_{L R}^{V_{o}}$ & $\checkmark$ & $\checkmark$ & $\times$ & $\times$ \\
\hline$Q_{R R}^{V_{o}}$ & $\checkmark$ & $\checkmark$ & $\times$ & $\times$ \\
\hline$Q_{11}^{V_{o}}$ & $\checkmark$ & $\checkmark$ & $\times$ & $\times$ \\
\hline$Q_{15}^{V_{o}}$ & $\checkmark$ & $\checkmark$ & $\times$ & $\times$ \\
\hline$Q_{55}^{V_{o}}$ & $\checkmark$ & $\checkmark$ & $\times$ & $\times$ \\
\hline \hline & & & \\
\hline
\end{tabular}

Table 2: Allowed $(\checkmark)$ and Disallowed $(\times)$ operators for singlet and octet scalar $\left(S_{s}, S_{o}\right)$, and, vector $\left(V_{s}, V_{o}\right)$ cases for various observables. The allowed region should satisfy the following constraints on the Tevatron and LHC observables: (1) $\left|\frac{\delta \sigma_{t \bar{t}}}{\sigma_{t \bar{t}}^{S M}}\right|<0.3$, where $\delta \sigma_{t \bar{t}}=\sigma_{t \bar{t}}^{\text {Total }}-\sigma_{t \bar{t}}^{S M}$ measured difference in the $t \bar{t}$ cross-section at the Teavtron, (2) $\chi_{A_{F B}^{t, l o w}}<2$, (3) $\chi_{A_{F B}^{t, h i g h}}<2$ that means the $\chi^{2}<2$ for the asymmetry in the low and in the high mass region from experiment; and, (4) $\sigma_{l^{ \pm} l^{ \pm}}<1 \mathrm{fb}$ with $l^{ \pm}=e^{ \pm}$ and $\mu^{ \pm}, \sigma_{l^{ \pm} l^{ \pm}}$is the same-sign dilepton cross-section for the LHC at $\sqrt{s}=7 \mathrm{TeV}$. The analysed ranges for exchanged particle mass, and the couplings are: $M_{X} \in[0.25,4] \mathrm{TeV}$, and $g_{X} \in[0.01,1]$. 


\subsection{SM background}

We now would like to address the SM background that could possibly confuse the aforementioned signal. The most serious contribution to SM background for this process is due to the production of $p p \longrightarrow W^{+} j W^{+} j$, where $W^{+} \longrightarrow l^{+} \nu_{l}$. Using MadGraph5, we estimate the bare cross-section for the final process $p p \longrightarrow W^{+} j W^{+} j \longrightarrow\left(l^{+} \nu_{l}\right) j\left(l^{+} \nu_{l}\right) j$ to be of about $3.66 \mathrm{fb}$ for the $\sqrt{s}=7 \mathrm{TeV}$ at the LHC, which reduces to $1.53 \mathrm{fb}$ when we demand the following basic cuts on the leptons and the jets: $p_{T_{l, j}}>25 \mathrm{GeV},\left|\eta_{l, j}\right| \leq 2.7$; $\Delta R_{l, l}, \Delta R_{l, j} \geq 0.4$ and $E_{T}>30 \mathrm{GeV}$.

The above SM background final-state topology can also be obtained when two partons from the same proton scatter into a $W^{+}$and a jet and a the same time partons from the other proton also scattering into another $W^{+} j$. This is known as Double-parton scattering (DPS) and its cross-section can be roughly estimated by [14],

$$
\sigma^{D P S}\left(W^{+} j W^{+} j\right)=\frac{\sigma\left(W^{+} j\right)^{2}}{2 \sigma^{e f f}}
$$

where, $\sigma^{e f f .}$ is the total effective cross-section at the LHC which has been measured to be about $11 \mathrm{mb}$ according to [15]. Using the above formula, we estimate, $\sigma^{D P S}\left(W^{+} j W^{+} j\right)$ $=0.9 \mathrm{fb}$ before any cuts which becomes $0.02 \mathrm{fb}$ when we allow both the W's leptonically and fold-in the basic cuts as discussed above.

The final contribution to the above background is due to the pile-up at the LHC which can be estimated using the following formula,

$$
\sigma^{\text {Pile }}\left(W^{+} j W^{+} j\right)=\frac{\sigma\left(W^{+} j\right)^{2}}{2 \sigma^{\text {inel. }}} N_{p}
$$

where $\sigma^{\text {inel. }}$ is the total inelastic cross-section at the LHC, and, $N_{p}$ is the number of pileup events per bunch-crossing which are measured to be $110 \mathrm{mb}$ and 32 respectively. [16]. We therefore obtain $\sigma^{\text {Pile }}\left(W^{+} j W^{+} j\right)=2.88 \mathrm{fb}$ without any cuts which translates into $0.06 \mathrm{fb}$ same-sign lepton events. Thus the total background cross-section for the same-sign lepton has been estimated to be about $1.53+0.02+0.06=1.61 \mathrm{fb}$.

Now because LHC did not observe such events, in order to apply the constraint due to such a process, we demand signal significance for this process to be less than 1 for the $5 \mathrm{fb}^{-1}$ data which is equivalent to having less than 8 signal events for the $5 \mathrm{fb}^{-1}$ assuming the events are distributed according to Poisson distribution.

For our analysis of same-sign lepton pairs, we first produce the same-sign top pairs using MadGraph and then allow them to decay semi-leptonically into a b-jet and a $\mu^{ \pm}$or $e^{ \pm}$ via a $W^{ \pm}$. We then impose the same basic cuts as mentioned before and finally demand 8 or lesser same-sign lepton pair events for $\int \mathcal{L} d t=5 f b^{-1}$ integrated LHC-7 luminosity. We found that for $M_{X}=500 \mathrm{GeV}$, these basic cuts reduce the same-sign lepton event rate by about $\epsilon_{C}=42 \%$. Now since each top-quark decays into a lepton about $25 \%$ of the time times when incorporating decays of leptonic decays of taus, therfore the effective event rate for the same-sign lepton pair would be $\sigma_{l^{ \pm} l^{ \pm}}=\sigma_{t t} \times B r(t \rightarrow b+l+\ldots)^{2} \times \epsilon_{C} \times \epsilon_{b}^{2} \simeq 0.01 \sigma_{t t}$, 


\begin{tabular}{|l|l|l|l|l|}
\hline \hline Operator & \multicolumn{5}{|c|}{$M_{X}(\mathrm{TeV})$} \\
\hline & 0.25 & 0.5 & 1 & 2 \\
\hline \multicolumn{5}{|c|}{ Case 1: Singlet Scalar } \\
\hline \hline$Q_{L L / R R}^{S_{s}}$ & 0.1 & 0.3 & 0.5 & 1 \\
\hline$Q_{L R}^{S_{s}}$ & 0.3 & 0.5 & 0.7 & 1 \\
\hline$Q_{11 / 55}^{S_{s}}$ & 0.3 & 0.5 & 0.7 & 1 \\
\hline$Q_{15}^{S_{s}}$ & 0.3 & 0.5 & 0.9 & 1 \\
\hline \hline \multicolumn{5}{|c|}{ Case 2: Octet Scalar } \\
\hline \hline$Q_{L L / R R}^{S_{o}}$ & 0.3 & 0.5 & 0.8 & 1 \\
\hline$Q_{L R}^{S_{o}}$ & 0.3 & 0.5 & 0.9 & 1 \\
\hline$Q_{11 / 55}^{S_{o}}$ & 0.5 & 0.7 & 0.8 & 1 \\
\hline$Q_{15}^{S_{o}}$ & 0.7 & 0.9 & 1 & 1 \\
\hline \hline \multicolumn{5}{|c|}{ Case 3: Singlet Vector } \\
\hline \hline$Q_{L L / R R}^{V_{s}}$ & 0.05 & 0.1 & 0.3 & 0.5 \\
\hline$Q_{L R}^{V_{s}}$ & 0.1 & 0.1 & 0.3 & 0.7 \\
\hline$Q_{11 / 55}^{V_{s}}$ & 0.1 & 0.1 & 0.3 & 0.7 \\
\hline$Q_{15}^{V_{s}}$ & 0.1 & 0.3 & 0.4 & 0.7 \\
\hline \hline \multicolumn{5}{|c|}{ Case 4: Octet Vector } \\
\hline \hline$Q_{L L / L L}^{V_{o}}$ & 0.1 & 0.1 & 0.3 & 0.6 \\
\hline$Q_{L R}^{V_{o}}$ & 0.1 & 0.3 & 0.7 & 1 \\
\hline$Q_{11 / 55}^{V_{o}}$ & 0.1 & 0.3 & 0.7 & 1 \\
\hline$Q_{15}^{V_{o}}$ & 0.25 & 0.6 & 0.7 & 1 \\
\hline \hline \multicolumn{5}{|c|}{ Oct } \\
\hline \hline
\end{tabular}

Table 3: Upper bounds on the coupling $g_{X}$ for various operators after demanding that the $t \bar{t}$ crosssection must not deviate from experiment by more than $10 \%$ and the events with same-sign lepton pair are consistent with the SM background at the $\mathrm{LHC}$ at $\sqrt{s}=7 \mathrm{TeV}$ and at integrated luminosity, $\int \mathcal{L} d t=5 \mathrm{fb}^{-1}$.

where, $\epsilon_{b}$, the b-tagging efficiency has been assumed to be $\sim 58 \%$ at the LHC [17]. Thus the observed $\sigma_{l^{ \pm} l^{ \pm}}$is only about 1 percent of the raw $\sigma_{t t}$.

\section{Conclusions}

We conclude our findings in Table 2 and 3 for all the cases. Table 2 shows that although all the operators are allowed by the $t \bar{t}$ cross-section data and by $\mathcal{A}_{F B}^{t, l o w}$, most of them are ruled out by $\mathcal{A}_{F B}^{t, h i g h}$ at the $2 \sigma$ (or equivalently $95 \%$ confidence level). Out of all the cases with scalar, vector and tensor operators, only two cases with a scalar singlet operators, $Q_{L L}^{S_{s}}$ and $Q_{R R}^{S_{s}}$, four cases with a singlet vector operators, $Q_{L L}^{V_{s}}, Q_{R R}^{V_{s}}, Q_{11}^{V_{s}}$, and $Q_{55}^{V_{s}}$ are not ruled out by the Tevatron data. Later these also get excluded by the imposition of the 
LHC constraint on the $\sigma_{l^{ \pm} l^{ \pm}}$. We are thus left with no allowed case by all four observables we analysed. This suggests that it is extremely difficult if not impossible, to provide a tree-level solution to the $\mathcal{A}_{F B}^{t}$ on the basis of pure (self-conjugate) flavor-changing top interactions.

Finally we also checked the viability of each of the operators when imposing only the total $t \bar{t}$ cross section and the same sign top cross-section, i.e. suppose we drop the $\mathcal{A}_{F B}^{t}$ constraints altogether assuming that $\mathcal{A}_{F B}^{t}$ is not a genuine effect due to new physics ${ }^{1}$. For this purpose we assume that the top-cross-section measurement has only $10 \%$ errors. Table 3 summarizes the resulting upper bounds on the $g_{X}$ for a wide range of $M_{X}$ for various operators. Clearly this suggest that bounds due to non-observation of same-sign leptons at the LHC are not as strong as the one which arise due to the imposition of $\mathcal{A}_{F B}$ constraints.

Thus, using an extremely general phenomenological Lagrangian we have shown that all models with t-channel self-conjugate flavor-changing resonance up to a scale of $4 \mathrm{TeV}$, that have been proposed to account for $\mathcal{A}_{F B}^{t}$, are ruled out. Since tree-level flavor-changing operators seem unable to account for the forward-backward top-quark production asymmetries and stay simultaneously consistent with the same-sign top-quark production data, if the experimental deviations of the forward-backward asymmetry from the Standard Model gets firmly established, one may need to resort to flavor-changing loop level new physics interactions; an example of this is studied in [18].

\section{Acknowledgements}

We thank Alex Kagan for a useful communication. The work of D. A. and A. S. are supported in part by US DOE grant Nos. DE-FG02-94ER40817 (ISU) and DE-AC02$98 \mathrm{CH} 10886$ (BNL). The work of S. K. G. was supported in part by the ARC Centre of Excellence for Particle Physics at the Tera-scale. The use of Monash University Sun Grid, a high-performance computing facility, is gratefully acknowledged.

\section{References}

[1] V. M. Abazov et al. [D0 Collaboration], Phys. Rev. D 84, 112005 (2011) [arxiv:1107.4995 [hep-ex]].

[2] T. Aaltonen et al. [CDF Collaboration], Phys. Rev. D 83, 112003 (2011) [arxiv:1101.0034 [hep-ex]].

[3] J. H. Kuhn and G. Rodrigo, Phys. Rev. Lett. 81, 49 (1998) [hep-ph/9802268]; J. H. Kuhn and G. Rodrigo, Phys. Rev. D 59, 054017 (1999) [hep-ph/9807420]; M. T. Bowen, S. D. Ellis and D. Rainwater, Phys. Rev. D 73, 014008 (2006) [hep-ph/0509267]; O. Antunano, J. H. Kuhn and G. Rodrigo, Phys. Rev. D 77, 014003 (2008) [arxiv:0709.1652 [hep-ph]]; S. Dittmaier, P. Uwer and S. Weinzierl, Phys. Rev. Lett. 98, 262002 (2007) [hep-ph/0703120 [HEP-PH]]; L. G. Almeida, G. F. Sterman and W. Vogelsang, Phys. Rev. D 78, 014008 (2008) [arxiv:0805.1885 [hep-ph]]; K. Melnikov and M. Schulze, Nucl. Phys. B 840, 129

\footnotetext{
${ }^{1}$ We thank Sally Dawson for this nice idea.
} 
(2010) [arxiv:1004.3284 [hep-ph]]; W. Hollik and D. Pagani, Phys. Rev. D 84, 093003 (2011) [arxiv:1107.2606 [hep-ph]]; J. H. Kuhn and G. Rodrigo, JHEP 1201, 063 (2012) [arxiv:1109.6830 [hep-ph]].

[4] V. Ahrens, A. Ferroglia, M. Neubert, B. D. Pecjak and L. L. Yang, JHEP 1009, 097 (2010) [arxiv:1003.5827 [hep-ph]].

[5] D. Choudhury, R. M. Godbole, R. K. Singh and K. Wagh, Phys. Lett. B 657, 69 (2007) [arxiv:0705.1499 [hep-ph]]; S. Jung, H. Murayama, A. Pierce and J. D. Wells, Phys. Rev. D 81, 015004 (2010) [arxiv:0907.4112 [hep-ph]]; K. Cheung, W. Y. Keung and T. C. Yuan, Phys. Lett. B 682, 287 (2009) [arxiv:0908.2589 [hep-ph]]; J. Shu, T. M. P. Tait and K. Wang, Phys. Rev. D 81, 034012 (2010) [arxiv:0911.3237 [hep-ph]]; A. Arhrib, R. Benbrik and C. H. Chen, Phys. Rev. D 82, 034034 (2010) [arxiv:0911.4875 [hep-ph]]; I. Dorsner, S. Fajfer, J. F. Kamenik and N. Kosnik, Phys. Rev. D 81, 055009 (2010) [arxiv:0912.0972 [hep-ph]]; V. Barger, W. Y. Keung and C. T. Yu, Phys. Rev. D 81, 113009 (2010) [arxiv:1002.1048 [hep-ph]]; B. Xiao, Y. k. Wang and S. h. Zhu, Phys. Rev. D 82, 034026 (2010) [arxiv:1006.2510 [hep-ph]]; D. W. Jung, P. Ko and J. S. Lee, Phys. Lett. B 701, 248 (2011) [arxiv:1011.5976 [hep-ph]]; K. Cheung and T. C. Yuan, Phys. Rev. D 83, 074006 (2011) [arxiv:1101.1445 [hep-ph]]; M. I. Gresham, I. W. Kim and K. M. Zurek, Phys. Rev. D 84, 034025 (2011) [arxiv:1102.0018 [hep-ph]]; V. Barger, W. Y. Keung and C. T. Yu, Phys. Lett. B 698, 243 (2011) [arxiv:1102.0279 [hep-ph]]; B. Bhattacherjee, S. S. Biswal and D. Ghosh, Phys. Rev. D 83, 091501 (2011) [arxiv:1102.0545 [hep-ph]]; B. Grinstein, A. L. Kagan, M. Trott and J. Zupan, Phys. Rev. Lett. 107, 012002 (2011) [arxiv:1102.3374 [hep-ph]]; K. M. Patel and P. Sharma, JHEP 1104, 085 (2011) [arXiv:1102.4736 [hep-ph]]; G. Isidori and J. F. Kamenik, Phys. Lett. B 700, 145 (2011) [arxiv:1103.0016 [hep-ph]]; A. R. Zerwekh, Phys. Lett. B 704, 62 (2011) [arxiv:1103.0956 [hep-ph]]; E. R. Barreto, Y. A. Coutinho and J. Sa Borges, Phys. Rev. D 83, 054006 (2011) [arxiv:1103.1266 [hep-ph]]; R. Foot, Phys. Rev. D 83, 114013 (2011) [arxiv:1103.1940 [hep-ph]]; Z. Ligeti, G. M. Tavares and M. Schmaltz, JHEP 1106, 109 (2011) [arxiv:1103.2757 [hep-ph]]; J. A. Aguilar-Saavedra and M. Perez-Victoria, JHEP 1105, 034 (2011) [arxiv:1103.2765 [hep-ph]]; M. I. Gresham, I. W. Kim and K. M. Zurek, Phys. Rev. D 83, 114027 (2011) [arxiv:1103.3501 [hep-ph]]; J. Shu, K. Wang and G. Zhu, Phys. Rev. D 85, 034008 (2012) [arxiv:1104.0083 [hep-ph]]; A. E. Nelson, T. Okui and T. S. Roy, Phys. Rev. D 84, 094007 (2011) [arxiv:1104.2030 [hep-ph]]; S. Jung, A. Pierce and J. D. Wells, Phys. Rev. D 84, 055018 (2011) [arxiv:1104.3139 [hep-ph]]; G. Zhu, Phys. Lett. B 703, 142 (2011) [arxiv:1104.3227 [hep-ph]]; D. W. Jung, P. Ko and J. S. Lee, Phys. Rev. D 84, 055027 (2011) [arxiv:1104.4443 [hep-ph]]; K. S. Babu, M. Frank and S. K. Rai, Phys. Rev. Lett. 107, 061802 (2011) [arxiv:1104.4782 [hep-ph]]; D. Krohn, T. Liu, J. Shelton and L. T. Wang, Phys. Rev. D 84, 074034 (2011) [arxiv:1105.3743 [hep-ph]]; A. Hektor, G. Hutsi, M. Kadastik, K. Kannike, M. Raidal and D. M. Straub, Phys. Rev. D 84, 031701 (2011) [arxiv:1105.5644 [hep-ph]]; Y. Cui, Z. Han and M. D. Schwartz, JHEP 1107, 127 (2011) [arxiv:1106.3086 [hep-ph]]; E. Gabrielli and M. Raidal, Phys. Rev. D 84, 054017 (2011) [arxiv:1106.4553 [hep-ph]]; M. Duraisamy, A. Rashed and A. Datta, Phys. Rev. D 84, 054018 (2011) [arxiv:1106.5982 [hep-ph]]; J. A. Aguilar-Saavedra and M. Perez-Victoria, JHEP 1109, 097 (2011) [arxiv:1107.0841 [hep-ph]]; G. M. Tavares and M. Schmaltz, Phys. Rev. D 84, 054008 (2011) [arxiv:1107.0978 [hep-ph]]; L. Vecchi, JHEP 1110, 003 (2011) [arxiv:1107.2933 [hep-ph]]; D. Y. Shao, C. S. Li, J. Wang, J. Gao, H. Zhang and H. X. Zhu, Phys. Rev. D 84, 054016 (2011) [arxiv:1107.4012 [hep-ph]]. K. Blum, Y. Hochberg and Y. Nir, JHEP 1110, 124 (2011) [arxiv:1107.4350 [hep-ph]]; M. I. Gresham, I. W. Kim and K. M. Zurek, Phys. Rev. D 85, 014022 (2012) 
[arxiv:1107.4364 [hep-ph]]; P. Ko, Y. Omura and C. Yu, Phys. Rev. D 85, 115010 (2012) [arxiv:1108.0350 [hep-ph]]; M. Frank, A. Hayreter and I. Turan, Phys. Rev. D 84, 114007 (2011) [arxiv:1108.0998 [hep-ph]]; H. Davoudiasl, T. McElmurry and A. Soni, Phys. Rev. D 85, 054001 (2012) [arxiv:1108.1173 [hep-ph]]; S. Jung, A. Pierce and J. D. Wells, Phys. Rev. D 84, 091502 (2011) [arxiv:1108.1802 [hep-ph]]; P. Ko, Y. Omura and C. Yu, JHEP 1201, 147 (2012) [arxiv:1108.4005 [hep-ph]]; J. Y. Liu, Y. Tang and Y. L. Wu, J. Phys. G G 39, 055003 (2012) [arxiv:1108.5012 [hep-ph]]; K. Kolodziej, Phys. Lett. B 710, 671 (2012) [arxiv:1110.2103 [hep-ph]]; J. N. Ng and P. T. Winslow, JHEP 1202, 140 (2012) [arxiv:1110.5630 [hep-ph]]; K. Yan, J. Wang, D. Y. Shao and C. S. Li, Phys. Rev. D 85, 034020 (2012) [arxiv:1110.6684 [hep-ph]]; D. W. Jung, P. Ko and J. S. Lee, Phys. Lett. B 708, 157 (2012) [arxiv:1111.3180 [hep-ph]]; L. Wang, L. Wu and J. M. Yang, Phys. Rev. D 85, 075017 (2012) K. S. Babu, J. Julio and Y. Zhang, Nucl. Phys. B 858, 468 (2012) [arxiv:1111.5021 [hep-ph]]; P. Ko, Y. Omura and C. Yu, Nuovo Cim. C 035N3, 245 (2012) [arxiv:1201.1352 [hep-ph]]; E. L. Berger, Q. -H. Cao, C. -R. Chen, J. -H. Yu and H. Zhang, Phys. Rev. Lett. 108, 072002 (2012) [arXiv:1201.1790 [hep-ph]]; S. S. Biswal, S. Mitra, R. Santos, P. Sharma, R. K. Singh and M. Won, Phys. Rev. D 86, 014016 (2012) [arXiv:1201.3668 [hep-ph]]; P. Ko, [arxiv:1202.0367 [hep-ph]]; B. Grinstein, C. W. Murphy, D. Pirtskhalava and P. Uttayarat, JHEP 1208, 073 (2012) [arXiv:1203.2183 [hep-ph]]; C. Han, N. Liu, L. Wu and J. M. Yang, Phys. Lett. B 714, 295 (2012) [arxiv:1203.2321 [hep-ph]]; D. Duffty, Z. Sullivan and H. Zhang, Phys. Rev. D 85, 094027 (2012) [arxiv:1203.4489 [hep-ph]]; E. Alvarez and E. C. Leskow, Phys. Rev. D 86, 114034 (2012) [arXiv:1209.4354 [hep-ph]].

[6] D. W. Jung, P. Ko, J. S. Lee and S. h. Nam, Phys. Lett. B 691, 238 (2010) [arxiv:0912.1105 [hep-ph]]; J. Cao, Z. Heng, L. Wu and J. M. Yang, Phys. Rev. D 81, 014016 (2010) [arXiv:0912.1447 [hep-ph]]; Q. H. Cao, D. McKeen, J. L. Rosner, G. Shaughnessy and C. E. M. Wagner, Phys. Rev. D 81, 114004 (2010) [arxiv:1003.3461 [hep-ph]]; D. Choudhury, R. M. Godbole, S. D. Rindani and P. Saha, Phys. Rev. D 84, 014023 (2011) [arxiv:1012.4750 [hep-ph]]; E. L. Berger, Q. -H. Cao, C. -R. Chen and H. Zhang, arXiv:1209.4899 [hep-ph].

[7] J. M. Campbell and R. K. Ellis, Phys. Rev. D 60, 113006 (1999) [hep-ph/9905386].

[8] V. M. Abazov et al. [D0 Collaboration], Phys. Rev. D 80, 071102 (2009) [arxiv:0903.5525 [hep-ex]].

[9] https://indico.cern. ch/getFile.py/access? contribId=45\&sessionId=36\&resId= 0\&materialId=slides\&confId=144537; See also, ATLAS-CONF-2011-139; S. Chatrchyan et al. [CMS Collaboration], JHEP 1108, 005 (2011) [arXiv:1106.2142 [hep-ex]]; S. Chatrchyan et al. [CMS Collaboration], arXiv:1212.6194 [hep-ex].

[10] J. A. Aguilar-Saavedra and M. Perez-Victoria, Phys. Lett. B 701, 93 (2011) [arxiv:1104.1385 [hep-ph]]; C. Degrande et al., Phys. Lett. B 703, 306 (2011) [arxiv:1104.1798 [hep-ph]]. For constraints from SSTs, in the context of specific models, see also [19-21].

[11] T. Stelzer and W. F. Long, Comput. Phys. Commun. 81, 357 (1994) [arxiv:hep-ph/9401258]; J. Alwall et al., JHEP 0709, 028 (2007) [arxiv:0706.2334 [hep-ph]]; J. Alwall, M. Herquet, F. Maltoni, O. Mattelaer, T. Stelzer, JHEP 1106, 128 (2011) [arxiv:1106.0522 [hep-ph]].

[12] N. D. Christensen and C. Duhr, Comput. Phys. Commun. 180, 1614 (2009) [arxiv:0806.4194 [hep-ph]].

[13] J. Pumplin, D. R. Stump, J. Huston, H. L. Lai, P. M. Nadolsky and W. K. Tung, JHEP 0207, 012 (2002) [arxiv:hep-ph/0201195]; D. Stump et al., JHEP 0310046 (2003). 
[14] A. Kulesza and W. J. Stirling, Phys. Lett. B 475, 168 (2000) [hep-ph/9912232]; J. R. Gaunt, C. -H. Kom, A. Kulesza and W. J. Stirling, Eur. Phys. J. C 69, 53 (2010) [arXiv:1003.3953 [hep-ph]].

[15] http://indico.cern.ch/getFile.py/access?contribId=61\&sessionId=11\&resId= 0\&material Id=slides\&conf $\mathrm{Id}=27458$

[16] http://lhc-machine-outreach.web.cern.ch/lhc-machine-outreach/collisions.htm

[17] ATLAS: Detector and physics performance technical design report, Volume 1, p317-346, 1999. CERN-LHCC-99-14

[18] H. Davoudiasl, T. McElmurry and A. Soni, Phys. Rev. D 85, 054001 (2012) [arXiv:1108.1173 [hep-ph]].

[19] S. K. Gupta, arXiv:1011.4960 [hep-ph].

[20] J. Cao, L. Wang, L. Wu and J. M. Yang, Phys. Rev. D 84, 074001 (2011) [arXiv:1101.4456 [hep-ph]].

[21] E. L. Berger, Q. -H. Cao, C. -R. Chen, C. S. Li and H. Zhang, Phys. Rev. Lett. 106, 201801 (2011) [arXiv:1101.5625 [hep-ph]]. 

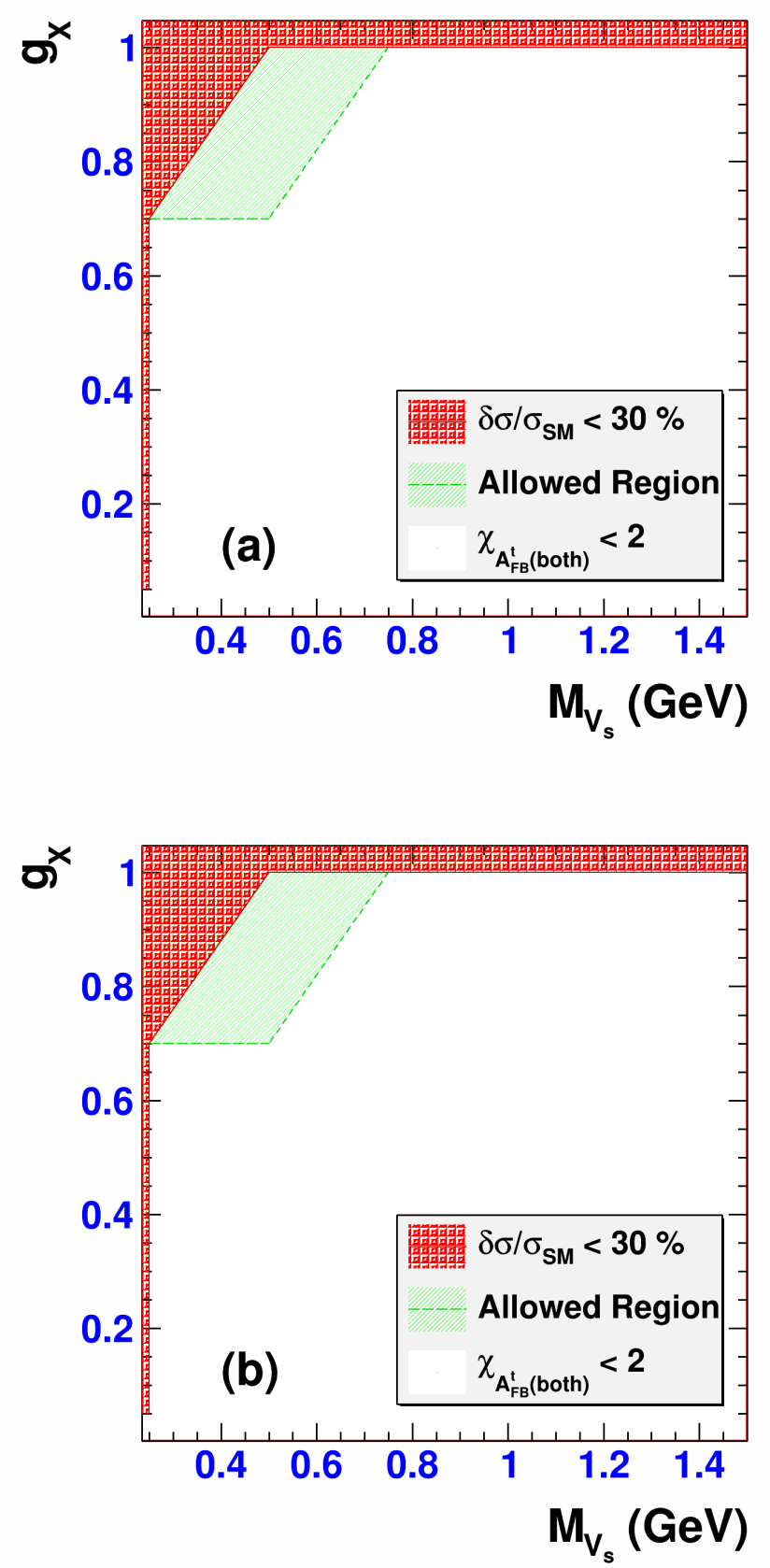

Figure 4: The allowed parameter space from the existing $t \bar{t}$ data in the $M$ versus $g_{X}$ plane is shown for the color singlet vector case. The couplings combinations considered are (a) 11, and (b) 55 (see Eqns. 1 for detail). The constraints imposed are that the low and the high mass integrated asymmetries, $\mathcal{A}_{F B}^{t, l o w}$ ad $\mathcal{A}_{F B}^{t, h i g h}$ remain within the $2 \sigma$ limits (symbolically labelled by $\chi_{A_{F B}^{t}}($ both $)<2$ ) from the experimental measurements (indicated by the region below the green dashed line) and the new physics contribution to the $t \bar{t}$ cross-section at the Teavtron is within $30 \%$ of the SM $t \bar{t}$ (the square hatched region above the red solid line). The diagonal relation between these two lines is thus the region which is allowed by these constraints. 

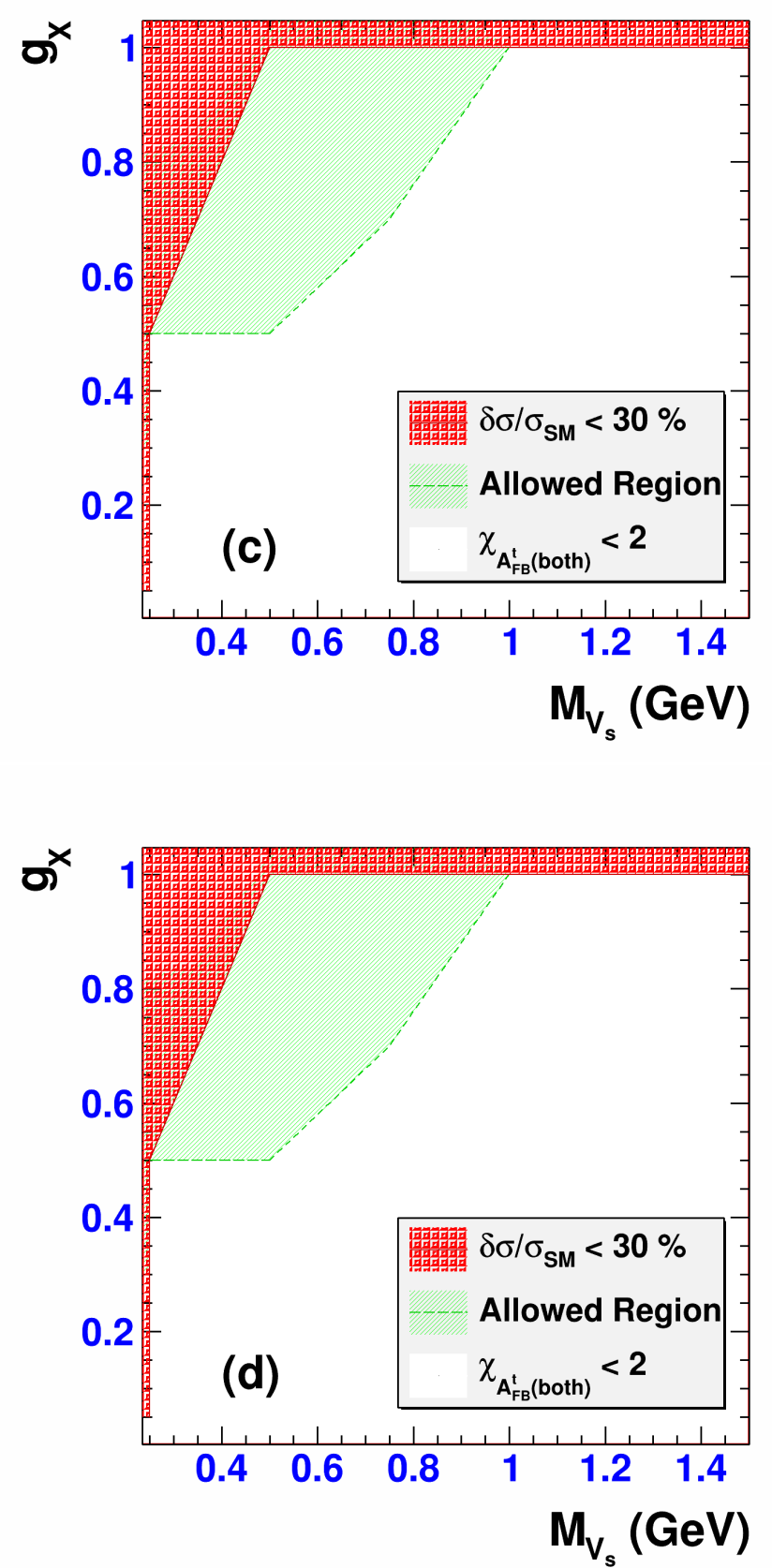

Figure 5: The exclusion plots here are similar to those of Figure 4 except that the vector couplings are (c) LL and (d) RR. 